\title{
Solvability of a Spatial Nonlinear Boundary Value Problem for Elastic Inhomogeneous Isotropic Ellipsoid
}

\author{
Gulnara A. Yakupova \\ Kazan Federal University \\ Email: gulnara_yakupova_2016@mail.ru
}

\section{Received: 02 ${ }^{\text {nd }}$ November 2018, Accepted: $2^{\text {th }}$ November 2018, Published: 31 $^{\text {st }}$ December 2018}

\begin{abstract}
The work is devoted to the proof of the existence theorem and the development of analytical numerical methods for finding solutions of geometrically nonlinear boundary value problems of the three-dimensional elasticity theory. Currently, the solvability of nonlinear spatial boundary-value problems for isotropic homogeneous and piecewisehomogeneous elastic bodies is most fully studied. Therefore, the development of mathematical methods to investigate the solvability and prove theorems on the existence of solutions of spatial nonlinear problems for anisotropic inhomogeneous elastic bodies is very important. In this paper, we study the solvability of nonlinear boundary value problems of the threedimensional elasticity theory for an isotropic inhomogeneous ellipsoid under kinematic boundary conditions. In the case of three-dimensional problems, the proposed research is based on integral approximations for displacements and on the fundamental solutions of Laplace, with which equilibrium equations are reduced to a system of three-dimensional singular integral equations in the volume occupied by an elastic body. The solvability of the system of integral equations is established using topological methods. Representations for displacements constructed in this way allow us to reduce the original system of equilibrium equations to a single nonlinear operator equation, the solvability of which is investigated using the principle of contracted mappings.
\end{abstract}

\section{Keywords}

Elastic Inhomogeneous Isotropic Body, Equilibrium Equations, Boundary Value Problem, Three-Dimensional Singular Integral Equations, Symbol of a Singular Operator, Existence Theorem

\section{Introduction}

When creating computer programs that allow solving complex problems of calculating elastic structures, the problem of adequacy of the model chosen to real processes comes to the fore. The solution to this problem is based on a rigorous mathematical study of the solvability of boundary value problems. Availability of existence theorems makes it easy to prove the convergence of numerical methods to the exact real solution. Therefore, a rigorous study of the solvability of boundary value problems and the proof of existence theorems are a very topical problem in the mathematical theory of elasticity. In this paper, the method is used based on application of integral approximations for the components of displacements to study the solvability of nonlinear boundary problems for an isotropic inhomogeneous elastic ellipsoid. The problem is reduced to a system of singular spherical integral equations, the solvability of which is established with the involvement of a singular operator.

\section{Methods}

The method proposed for studying the solvability of spatial nonlinear boundary value problems is based on integral approximations for displacements. Here, they are constructed using an approach based on the use of a harmonic Green function and the Dirichlet problem in the case of elastic bodies of special configuration (ball, half-space, cylinder, etc.) and the harmonic potential theory for the case of certain bones elastic bodies. A distinctive feature of the proposed method is that the fundamental solutions underlying the potential theory are not related to the original system of equilibrium equations, they are only solutions of the Laplace equation. The integral approximations obtained in this way define displacements satisfying the given boundary conditions and the Poisson equation with an arbitrary right-hand side. The equilibrium equations are satisfied by choosing the right-hand side of the Poisson equation, for the determination of which a system of nonlinear three-dimensional singular integral equations is derived equivalent to the original system of equilibrium equations in terms of the volume occupied by an elastic body. The theory of multidimensional integral equations developed by Professor S.G. Mikhailin is used to study the solvability of a system of integral equations [1].

\section{Results and Discussion}

There is considered a system of nonlinear equations of the form:

$$
\sigma_{, j}^{k j}+f_{k}+X_{k}=0, k=\overline{1,3}
$$

In the ellipsoid $\mathrm{V}$ centered at the coordinate origin and having the boundary $\partial V: \frac{x_{1}^{2}}{a^{2}}+\frac{x_{2}^{2}}{b^{2}}+\frac{x_{3}^{2}}{c^{2}}=1$; (hereinafter, the values with recurring Latin indices are summed starting from 1 to 3 ), where the following notation is adopted:

$$
\begin{aligned}
f_{1}=\frac{\partial}{\partial x_{j}}\left(\sigma^{j 3} \omega_{2}-\sigma^{j 2} \omega_{3}\right), f_{2} & =\frac{\partial}{\partial x_{j}}\left(\sigma^{j 1} \omega_{3}-\sigma^{j 3} \omega_{1}\right), f_{3}=\frac{\partial}{\partial x_{j}}\left(\sigma^{j 2} \omega_{1}-\sigma^{j 1} \omega_{2}\right) ; \\
\sigma^{j j}=2 \varepsilon_{j j}+\lambda \varepsilon, \sigma^{j k} & \equiv \sigma^{k j}=\mu \varepsilon_{j k}, j \neq k ; \varepsilon=\varepsilon_{11}+\varepsilon_{22}+\varepsilon_{33} ;
\end{aligned}
$$




$$
\begin{gathered}
\varepsilon_{k j}=e_{k j}+æ_{k j}, e_{j j}=u_{j, j}, e_{j k}=u_{j, k}+u_{k, j}, æ_{j j}=\left(\omega_{1}^{2}+\omega_{2}^{2}+\omega_{3}^{2}-\omega_{j}^{2}\right) / 2, \\
æ_{k j}=-\omega_{k} \omega_{j}, j \neq k, j, k=\overline{1,3} ; \omega_{1}=\left(u_{3,2}-u_{2,3}\right) / 2, \\
\omega_{2}=\frac{\left(u_{1,3}-u_{3,1}\right)}{2}, \omega_{3}=\frac{\left(u_{2,1}-u_{1,2}\right)}{2} ; \mu=\frac{E}{2(1+v)}, \lambda=\frac{v E}{(1+v)(1-2 v)}
\end{gathered}
$$

And the symbol $\sigma_{, j}^{k j}$ in (1) means the partial derivative $\sigma_{, j}^{k j} \equiv \partial \sigma^{k j} / \partial x_{j}$..

The system of equations (1) together with relations (2) describes the equilibrium state of an elastic isotropic inhomogeneous ellipsoid [2, pp.83-84]. Upon that: $\sigma^{k j}=\sigma^{j k}$ - the stress components $\varepsilon_{k j}=\varepsilon_{j k}-$ the components of deformations, $\omega_{k}$-rotation angles of an element around the axis $O x_{k}, u=\left(u_{1}, u_{2}, u_{3}\right)$ - the displacement vector), $u_{j, k} \equiv$ $\partial u_{j} / \partial x_{k}, j, k=\overline{1,3} ; X_{k}(k=\overline{1,3})$ - the components of the volumetric external forces applied to an elastic body; $\mu$ - shear modulus of elasticity, $\lambda$ - Lame parameter, $E=E(x)$ - tensile elasticity modulus, $v=v(x)$ - Poisson's ratio, $x=\left(x_{1}, x_{2}, x_{3}\right)$ - rectangular Cartesian coordinates of the body's point in the region $\mathrm{V}$.

If in the system (1) the stresses and strains are replaced by their expressions from (2), then we obtain a system of equilibrium equations expressed in displacements:

$$
\Delta u_{k}+\theta_{, k} /(1-2 v)+l_{k}(u)+g_{k}(u)+X_{k} / \mu=0, k=\overline{1,3},
$$

Where

$$
\begin{aligned}
& l_{k}(u)=\left[\mu_{, k} e_{k k}+\mu_{, j} e_{k j}+\lambda_{, k}\left(e_{11}+e_{22}+e_{33}\right)\right] / \mu, \\
& g_{k}(u)=\frac{1}{\mu}\left\{f_{k}(u)+\frac{\partial}{\partial x_{k}}\left[(\mu+\lambda)\left(\chi_{11}+\chi_{22}+\chi_{33}\right)\right]+\frac{\partial}{\partial x_{j}}\left(\mu \chi_{j k}\right)\right\}, \theta=\operatorname{div} u ;
\end{aligned}
$$

$\Delta$ - Laplace operator.

Problem I. It is required to find a solution $u=\left(u_{1}, u_{2}, u_{3}\right)$ for the system (1) in the field $\mathrm{V}$ satisfying on its border $\partial \mathrm{V}$ to the condition

$$
u=0
$$

Problem I will be studied in a generalized formulation. Let the following conditions be fulfilled: (a) $E(x), v(x) \in$ $W_{p}^{(1)}(V), p>3$; (b) $X_{k} \in L_{p}(V), p>3, k=\overline{1,3}$

Definition: We call the displacement vector $u=\left(u_{1}, u_{2}, u_{3}\right) \in W_{p}^{(2)}(V), p>3$, almost everywhere satisfying system (1) and the boundary condition (4) as the generalized solution of the problem I.

Here $\mathrm{W}_{\mathrm{p}}^{(\mathrm{j})}(\mathrm{V})(\mathrm{j}=1,2)$ is Sobolev space. By virtue of embedding theorems for Sobolev spaces $\mathrm{W}_{\mathrm{p}}^{(\mathrm{j})}(\mathrm{V})$ with $p>3$ the generalized decision $u \in C_{\alpha}^{1}(\bar{V})$, and elastic characteristics $B^{j k n m} \in C_{\alpha}(\bar{V}), \alpha=(p-3) / p, p>3$.

To treat the problem I, we use the method developed in [3-5], where linear spatial problems for a homogeneous isotropic ball [3], certain anisotropic inhomogeneous body [4], and nonlinear boundary problems for a non-uniform isotropic ball [5] have been treated.

Let $y=\varphi(x)=\left(\varphi_{1}(x), \varphi_{2}(x), \varphi_{3}(x)\right)\left(y=\left(y_{1}, y_{2}, y_{3}\right)\right)$ be one-to-one mapping of the area $\mathrm{V}$ on the ball $S_{R}: y_{1}^{2}+y_{2}^{2}+$ $y_{3}^{2} \leq R^{2}$. Using $x=\psi(y)=\left(\psi_{1}(y), \psi_{2}(y), \psi_{3}(y)\right)$ we denote the mapping which is inverse in relation to $y=\varphi(x)$ .We will assume that $\varphi(x) \in W_{p}^{(2)}(V), \psi(y) \in W_{p}^{(2)}\left(S_{R}\right), p>3$.

the form

In (3), we proceed to the new variables $y_{j}, j=\overline{1,3}$. Then in the ball $S_{R}$ we get the system of equations having

Where

$$
a_{k j}^{n m} u_{j y_{n} y_{m}}+l_{k}(u)+g_{k}(u)+X_{k}=0, k=\overline{1,3}
$$

$$
\begin{aligned}
& l_{k}(u)=\mu\left[u_{k y_{n}} 1^{j} \varphi_{n x_{j} x_{j}}+u_{j y_{n}} \varphi_{n x_{k} x_{j}}\right]+e_{k k}(x) \mu_{y_{n}} \varphi_{n x_{k}}+e_{k j}(x) \mu_{y_{n}} \varphi_{n x_{j}}+1^{j} e_{j j}(x) \lambda_{y_{n}} \varphi_{n x_{k}}, \\
& g_{k}(u)=f_{k}(u)+\left(\mu_{y_{n}}+\lambda_{y_{n}}\right) \varphi_{n x_{k}} 1^{j} \chi_{j j y_{n}}(x) \varphi_{n x_{k}}+\mu_{y_{n}} \varphi_{n x_{j}} \chi_{j k y_{n}}(x) \varphi_{n x_{j}}, x=\psi(y), \\
& a_{k j}^{n m} \equiv a_{k j}^{n m}(y)=b_{k j}^{q s}(x) \varphi_{n x_{q}}(x) \varphi_{m x_{s}}(x), \\
& b_{j j}^{j j}=2 \mu+\lambda \equiv \alpha, b_{j j}^{k k}=\mu(j \neq k), b_{n m}^{n m}=\mu+\lambda \equiv \beta, k, j, n, m=\overline{1,3} ;
\end{aligned}
$$

The symbol $1^{j} \varphi_{n x_{j} x_{j}}$ means summation over $j: 1^{j} \varphi_{n x_{j} x_{j}}=\sum_{j=1}^{3} \varphi_{n x_{j} x_{j}}$.

Let's notice that $l_{k}(u), g_{k}(u)$ are linear completely continuous and nonlinear bounded operators in $L_{p}\left(S_{R}\right), p>3$ respectively

System (6) solution for the ball $S_{R}$ satisfying to the condition (4) on its boundary $\partial S_{R}: y_{1}^{2}+y_{2}^{2}+y_{3}^{2}=R^{2}$ will be searched as

$$
u(y)=\iiint_{S_{R}} G(x, y) \rho(x) d x, d x=d x_{1} d x_{2} d x_{3},
$$

Where $\rho=\left(\rho_{1}, \rho_{2}, \rho_{3}\right)$ is an arbitrary vector function belonging to the space $L_{p}\left(S_{R}\right), p>3 ; G(x, y)$ is a harmonic Green function of the Dirichlet problem for the ball $S_{R}$

The relation (8) is substituted in (6) and in order to determine the function $\rho=\left(\rho_{1}, \rho_{2}, \rho_{3}\right)$, we come to the system of three-dimensional nonlinear singular integral equations of the form

$$
P_{k j}\left(\rho_{j}\right)+g_{k}(\rho)+X_{k}=0, k=\overline{1,3}
$$


Where $\quad P_{k j}\left(\rho_{j}\right)=-\frac{1}{3}\left(a_{k j}^{11}+a_{k j}^{22}+a_{k j}^{33}\right) \rho_{j}(x)+\frac{1}{4 \pi} a_{k j}^{n m} \iiint_{E_{3}} \frac{f_{n m}(\theta)}{|x-y|^{3}} \rho_{j}^{*}(x) d x+l_{k}(\rho), f_{n m}(\theta)=3 \theta_{n} \theta_{m}-\delta_{n m}, \theta_{j}=$ $\left(x_{j}-y_{j}\right) /|x-y|, \theta=\left(\theta_{1}, \theta_{2}, \theta_{3}\right), \delta_{n m}=1$ at $n=m$ and $\delta_{n m}=0$ at $n \neq m ; n, m, k, j=\overline{1,3} ; \rho_{j}^{*}(x)=\rho_{j}(x)$ at $x \in S_{R}$ and $\rho_{j}^{*}(x)=-\left(R^{5} /|x|^{5}\right) \rho_{j}\left(R^{2} /|x|^{2}\right)$ at $x \bar{\epsilon} \overline{S_{R}}, E_{3}$ is a three-dimensional Euclidean space.

Note that $P_{k j}\left(\rho_{j}\right)$ are linear bounded operators in $L_{p}\left(S_{R}\right), p>3$ at their essence.

In studying the solvability of system (9), we follow [1]. Let denote the symbol of the singular operator $P_{k j}\left(\rho_{j}\right)$ with $\Phi_{k j}(y, \theta)$. We can show that the symbol $\Phi_{k j}(y, \theta)$ is given by the formula

Where

$$
\Phi_{k j}(y, \theta)=-b_{k j}^{n m}(x) \gamma_{n} \gamma_{m}, n, m, j, k=\overline{1,3},
$$

$$
\gamma_{n}=\varphi_{1 x_{n}}(x) \theta_{1}+\varphi_{2 x_{n}}(x) \theta_{2}+\varphi_{3 x_{n}}(x) \theta_{3}, n=\overline{1,3}, x=\psi(y), y \in S_{R}, x \in V
$$

Introducing (7) in (10), we will have

$$
\begin{gathered}
\Phi_{11}(y, \theta)=-\alpha \gamma_{1}^{2}-\mu \gamma_{2}^{2}-\mu \gamma_{3}^{2}, \Phi_{22}(y, \theta)=-\mu \gamma_{1}^{2}-\alpha \gamma_{2}^{2}-\mu \gamma_{3}^{2}, \\
\Phi_{33}(y, \theta)=-\mu \gamma_{1}^{2}-\mu \gamma_{2}^{2}-\alpha \gamma_{3}^{2}, \Phi_{j k}(y, \theta)=-2 \beta \gamma_{j} \gamma_{k}, j \neq k, j, k=\overline{1,3}
\end{gathered}
$$

We introduce determinants $\quad \Delta_{1}=\Phi_{11}(y, \theta), \quad \Delta_{2}=\operatorname{det}\left(\Phi_{k j}(y, \theta)\right)_{2 \times 2}, \quad \Delta_{3}=$ $\operatorname{det}\left(\Phi_{k j}(y, \theta)\right)_{3 \times 3}$. Then we get the following expressions for them:

$\Delta_{2}=\gamma_{1}^{2}\left[\mu \alpha \gamma_{1}^{2}+\mu_{0} \gamma_{2}^{2}+\mu(\mu+\alpha) \gamma_{3}^{2} / 2\right]+\gamma_{2}^{2}\left[\mu_{0} \gamma_{1}^{2}+\mu \alpha \gamma_{2}^{2}+\mu(\mu+\alpha) \gamma_{3}^{2} / 2\right]+$

$+\gamma_{3}^{2}\left[\mu(\mu+\alpha)\left(\gamma_{1}^{2}+\gamma_{2}^{2}\right) / 2+\mu^{2} \gamma_{3}^{2}\right]$,

$\Delta_{3}=-\left\{\gamma_{1}^{4}\left[\mu^{2} \alpha \gamma_{1}^{2}+\alpha_{0}\left(\gamma_{2}^{2}+\gamma_{3}^{2}\right)\right]+\gamma_{1}^{2} \gamma_{2}^{2}\left[\alpha_{0}\left(\gamma_{1}^{2}+\gamma_{2}^{2}\right)+\beta_{0} \gamma_{3}^{2}\right]+\right.$ $+\gamma_{1}^{2} \gamma_{3}^{2}\left[\alpha_{0}\left(\gamma_{1}^{2}+\gamma_{3}^{2}\right)+\beta_{0} \gamma_{2}^{2}\right]+\gamma_{2}^{4}\left[\mu^{2} \alpha \gamma_{2}^{2}+\alpha_{0}\left(\gamma_{1}^{2}+\gamma_{3}^{2}\right)\right]+$ $\left.+\gamma_{2}^{2} \gamma_{3}^{2}\left[\alpha_{0}\left(\gamma_{2}^{2}+\gamma_{3}^{2}\right)+\beta_{0} \gamma_{1}^{2}\right]+\gamma_{3}^{4}\left[\mu^{2} \alpha \gamma_{3}^{2}+\alpha_{0}\left(\gamma_{1}^{2}+\gamma_{2}^{2}\right)\right]\right\}$

Where

$$
\mu_{0}=\left(\mu^{2}+\alpha^{2}-4 \beta^{2}\right) / 2, \alpha_{0}=\mu\left(\mu \alpha+\mu_{0}\right) / 2, \beta_{0}=2\left(\mu^{3}+8 \beta^{3}-\alpha^{3}+3 \alpha \mu_{0}\right) / 3
$$

Let Poisson's Ratio $v=v(x)$ satisfies the condition

$$
-1<v(x) \leq v_{0}<1 / 2, \forall x \in \bar{V}, v_{0}=\text { const. }
$$

Then in the conditions of (a) and (12) functions will be the following $\alpha=\alpha(x), \mu=\mu(x) \geq c>0 \forall x \in \bar{V}$. In addition, by direct calculation we make sure that $\mu_{0}=\mu_{0}(x), \alpha_{0}=\alpha_{0}(x), \beta_{0}=\beta_{0}(x) \geq c>0 \forall x \in \bar{V}$ Then the expressions in square brackets in (11) are positive definite quadratic forms with respect to the variables $\gamma_{1}, \gamma_{2}, \gamma_{3}$ Therefore $\left|\Delta_{j}\right| \geq c>$ $0(j=\overline{1,3}) \forall y \in \overline{S_{R}}, \forall \theta \in \partial S_{1}$. As a result, the exact lower bounds of the moduli for the determinants $\Delta_{j}(j=\overline{1,3})$ are positive. Then [1, p. 192], the index of the system of equations (9) is zero and the Fredholm alternative is applicable to it. As a result, system (9) will be reduced to an equivalent system of the form

$$
\rho-G \rho=0
$$

Where $G \rho$ is a nonlinear bounded operator in $L_{p}\left(S_{R}\right), p>3$ and for any $\rho^{j}(j=1,2) \in L_{p}\left(S_{R}\right), p>3$ belonging to a ball $\left\|\rho^{j}\right\|_{L_{p}\left(S_{R}\right)}<r$ the assessment $\left\|G\left(\rho^{1}\right)-G\left(\rho^{2}\right)\right\|_{L_{p}\left(S_{R}\right)} \leq\left(q_{1}+q_{2} r\right) r\left\|\rho^{1}-\rho^{2}\right\|_{L_{p}\left(S_{R}\right)}$ is fair where $q_{j}(j=1,2)$ are known constants independent of $r$.

Let's suppose the radius $r$ of the ball and external forces applied to an elastic body are such that the conditions are met

$$
q=\left(q_{1}+q_{2} r\right) r<1,\|G(0)\|_{L_{p}\left(S_{R}\right)}<(1-q) r
$$

Under these conditions, the principle of contracted mappings [6, p.146] can be applied to equation (13) according to which equation (13) in a ball $\|\rho\|_{L_{p}\left(S_{R}\right)}<r$ has the only solution $\rho \in L_{p}\left(S_{R}\right), p>3$. Knowing $\rho=\left(\rho_{1}, \rho_{2}, \rho_{3}\right)$ and using the formula (8) we find the solution $u=\left(u_{1}, u_{2}, u_{3}\right) \in W_{p}^{(2)}\left(S_{R}\right), p>3$ of the Problem I.

Thus, the following main theorem is proved.

Theorem. Let the conditions (a), (b) and (5), and inequality (14) are satisfied. Then the Problem I for an elastic inhomogeneous isotropic ellipsoid has a unique generalized solution $u=\left(u_{1}, u_{2}, u_{3}\right) \in W_{p}^{(2)}(V), p>3$

\section{Summary}

Studies of the spatial boundary value problems solvability in the elasticity theory are conducted in two basic directions. The first direction is based on the application of functional analysis methods (the method of Hilbert spaces, variational methods, implicit function theorems) which allow us to investigate the existence of generalized solutions for a wide class of problems in the theory of elasticity in different energy spaces. Theorems on the existence of solutions of linear boundary value problems are proved when working out this direction (G. Fiker. Existence theorems in the theory of elasticity. - M.: Mir, 1974.-160 p.; G. Duvo, J.-L. Lions. Inequalities in mechanics and physics. - M.: Nauka, 1980.-384 p.), as well as nonlinear boundary value problems of the theory of elasticity (Sjärle F. Mathematical theory of elasticity, Translated from English - M.: Mir, 1992. - 472 p.). Investigations of the second direction are based on the theory of singular integral equations (Kupradze V.D., Hegelia T.G., Bashleyshvili M.O., Burguladze T.V. Three-dimensional problems in the mathematical theory of elasticity and thermoelasticity. -M.: Nauka, 1976.- 664 pp.) which are based on the fundamental solutions of equilibrium equations. At present, such fundamental solutions are constructed for equations with constant and piecewise-constant coefficients, describing the equilibrium state of isotropic homogeneous and piecewise- 
homogeneous elastic bodies. The study proposed within the framework of this work and relating to three-dimensional problems is the development of research in the second direction.

\section{Conclusion}

An existence theorem is proved and an analytical method is developed for finding solutions of geometrically nonlinear spatial boundary problems for an elastic isotropic inhomogeneous ellipsoid under kinematic boundary conditions.

Acknowledgements

The work is performed according to the Russian Government Program of Competitive Growth of Kazan Federal University.

\section{References}

[1] Mikhlin S.G. Multidimensional singular integrals and integral equations. - L.: Fizmatgiz, 1962. - 256 p.

[2] Novozhilov V.V. Fundamentals of the nonlinear theory of elasticity. - L.-M.: Gostekhizdat, 1948. - 576 p.

[3] Timergaliev S.N., Uglov A.N., Yakupova G.A. Investigation of the solvability of spatial boundary value problems for a ball in the linear theory of elasticity // Scientific and Technical Bulletin of the Volga region. - 2014. - №1.- P.41-44.

[4] Timergaliev S.N. On the solvability of boundary value problems for a system of differential equations of the threedimensional theory of elasticity // Proceedings of the Mathematical Center. N.I. Lobachevsky: materials of the International Scientific Conference "Boundary Value Problems for Differential Equations and Analytic Functions-2014". - Kazan: Kazan University Press, 2014. - V.49.- Pp.309-314.

[5] Timergaliev S.N., Yakupova G.A. On the existence of solutions of spatial nonlinear boundary value problems for an elastic inhomogeneous ball // Grid methods for boundary value problems and applications. Materials of the Tenth International Conference (Kazan, September 24-29, 2014). - Kazan: Kazan University Press, 2014. - P.586-591.

[6] Krasnoselsky M.A. Topological methods in the theory of nonlinear integral equations. - M. : Gostekhizdat, 1956. 392 p. 